\title{
Modelo teórico para diagnóstico da transparência em serviços: uma proposta para o setor de alimentos
}

\section{A theoretical model for transparency diagnoses in services: a proposal for the food sector}

\author{
Marcella Lomba Nicastro, Universidade Federal do Paraná. \\ marcellaln@gmail.com
}

Aguinaldo dos Santos, Universidade Federal do Paraná. asantos@ufpr.br

\section{Resumo}

A transparência é um dos princípios fundamentais para possibilitar o desenvolvimento sustentável, sendo necessária a comunicação plena das práticas sociais, ambientais e econômicas associadas às escolhas do consumidor. As tecnologias digitais expandiram as possibilidades de aumentar o nível de transparência em produtos e serviços. Embora a transparência tenha sido apontada como uma prioridade nas agendas de inovação, ainda há uma falta de compreensão do seu potencial de aplicação no Design de Serviço. Os autores investigaram o tema dentro do setor de alimentos, empregando como método a Design Science Research integrada à Revisão Bibliográfica, Estudo de Casos Múltiplos e Design Etnográfico. O principal resultado da pesquisa é a proposta de um modelo teórico para avaliar e incorporar a transparência rumo à sustentabilidade em serviços digitais no setor de alimentos.

Palavras-chave: Transparência, Design de Serviço, Serviço Digital, Sustentabilidade, Alimentação.

\begin{abstract}
Transparency is one of the fundamental principles to enable sustainable development, being necessary to fully communicate social, environmental, and economic practices associated with consumer's choices. Digital technologies have expanded the possibilities to increase the level of transparency across products and services. While transparency has been pointed to as a priority in innovation agendas, there is still a lack of understanding of the implications of applying it to Service Design, particularly in the food sector. The authors investigated the theme employing a Design Science Research integrated with a Literature Review, Multiple Case Studies, and Ethnographic Design Research. The research's main output is a theoretical model to assess and embed transparency towards sustainability on digital services in the food sector.
\end{abstract}

Keywords: Transparency, Service Design, Digital Service, Sustainability, Food. 


\section{Introdução}

Os produtos e serviços associados à alimentação estão em constante evolução em resposta à inovação de ordem tecnológica, às mudanças nos estilos de vida dos consumidores e, também, às alterações nos modelos de negócio das empresas operando neste setor. Dentre as mudanças contemporâneas no setor de alimentos está a crescente demanda, por parte dos consumidores, por maior controle, compreensão e participação nas decisões e práticas associadas à alimentação. Esta demanda tem sido impulsionada pela maior visibilidade e atenção do público quanto às práticas como contaminação por agrotóxicos, maus tratos e doenças provocadas em animais, fraudes, entre outros (WOGNUM et al., 2011; SCHIEFER; DEITERS, 2013).

Além dos fatores de segurança alimentar, a preocupação do consumidor tem evoluído para uma maior atenção às dimensões da sustentabilidade associadas aos alimentos, tais como conformidade ambiental, responsabilidade social e impacto econômico em toda a cadeia de valor (WOGNUM et al., 2011; SCHIEFER; DEITERS, 2013). Esta situação reforça a necessidade de disseminação das práticas associadas ao aumento da transparência.

A transparência é um dos princípios para se alcançar o desenvolvimento sustentável. Sua aplicação, em especial no setor de alimentos, pode permitir que tanto os consumidores quanto os demais stakeholders (como o produtor, distribuidor, varejo, governo, entre outros) participem de forma mais informada do sistema, favorecendo a qualidade da tomada de decisões e propiciando comportamentos de consumo em direção a padrões mais sustentáveis.

As iniciativas voltadas ao aumento da transparência na alimentação têm enfatizado tradicionalmente sua aplicação em nível de produto, por meio do projeto informacional dos atributos regulamentares que caracterizam o alimento (ex: ingredientes, valor nutricional, certificações, origem de produção, entre outros), combinados às ações de comunicação das marcas (SCHIEFER; DEITERS, 2013). Tais iniciativas permanecem relevantes; contudo, no contexto da emergência dos serviços digitais e da perspectiva sistêmica da transparência, não são mais suficientes para prover a transparência demandada pelos consumidores.

Ainda, tecnologias digitais (ex: aplicações mobile, internet, redes sociais, e-commerce, inteligência artificial, sensores etc.) têm propiciado formas radicalmente inovadoras de provimento e interação com a informação. Exemplos incluem aplicativos para compra de alimentos e para adequar preferências de consumo, dentre outros (POUTANEN et al., 2017).

No âmbito do Design, a transparência de serviços digitais associados à alimentação é um tema emergente que vem sendo tratado particularmente entre a comunidade atuante no Food Design (Design na Alimentação). O Food Design propõe aplicar conceitos, métodos e ferramentas para melhorar a relação dos consumidores com os alimentos em seus diversos sentidos e dimensões. Tal contribuição abrange desde o design do produto comestível e serviços associados até a configuração do sistema em seu entorno (COSTA; YUDI, 2017).

Para ampliar a transparência no setor de alimentos, é importante que o (a) Designer vá além da compreensão tão somente das necessidades e requisitos dos consumidores (BUCHANAN, 2001). O profissional, neste contexto, necessita considerar os requisitos da totalidade de stakeholders envolvidos. De fato, sob tal perspectiva é relevante compreender que a transparência 
tem implicações diretas como indutoras e balizadoras da sustentabilidade em todas as suas dimensões, incluindo os aspectos não antropocêntricos. Assim, para sua adequada implantação, é necessário integrar na práxis do Design os princípios e conceitos pertinentes a uma visão de sociedade com maior responsabilidade ambiental, maior equidade e coesão social, assim como maior justiça econômica.

Neste contexto, dada a importância da transparência na indução de comportamentos mais sustentáveis em serviços digitais associados aos alimentos, faz-se necessário o desenvolvimento de métodos, ferramentas, heurísticas e critérios de apoio ao processo de Design (SANGIORGI, et al., 2014; OSTROM et al., 2015). O presente artigo apresenta um estudo que contribui para o tema, com a proposição de um modelo de apoio ao Design de Serviço, tanto no diagnóstico como na criação de soluções voltadas à ampliação da transparência em serviços digitais do setor de alimentos.

\section{Transparência para o Design: serviços digitais na alimentação}

\subsection{Design de serviços digitais}

O Design de Serviço pode ser visto como uma abordagem centrada no usuário, implicando uma visão sistêmica sobre o processo de projeto. Envolve não somente o projeto da experiência do usuário, mas também o projeto do processo de negócio para que se possa entregar a experiência pretendida. Projetar para serviços ao invés de produtos significa que o que está sendo projetado é uma plataforma de ação, um sistema que possibilita a multiplicidade de interações (FREIRE; DAMAZIO, 2010). O Design de Serviço ajuda a projetar novos serviços ou aprimorar serviços existentes, para torná-los mais úteis, utilizáveis e desejáveis para os usuários, além de eficientes e eficazes para as organizações (MORITZ, 2005).

À medida que novas tecnologias são desenvolvidas e antigas são reconfiguradas ou suprimidas, novas formas e modalidades de serviços surgem com base em novos modelos de negócio e novas formas de interação. Este movimento, tanto impulsionado pelo usuário (user driven) como pela tecnologia (technology driven), transforma a forma como as inovações são conceituadas, projetadas, comercializadas e como as empresas as operam (OSTROM et al., 2015; PENIN, 2017; WEF, 2018).

O termo "serviço digital" ou serviço habilitado digitalmente (digital enabled services) vem sendo utilizado para se referir aos serviços baseados em tecnologias de informação e comunicação, onde o grau de dependência digital pode variar de acordo com o tipo de tecnologia e adoção pelo mercado e negócio (PENIN, 2017; HARTWIG; BILLERT, 2018). Os serviços digitais são caracterizados por uma interação direta do usuário com o serviço por meio de pontos de contatos digitais. O ponto de contato digital (que normalmente consiste em um website ou aplicativo com acesso à internet) serve como mediador da prestação do serviço, como, por exemplo, uma loja de compras online.

Dentre as características dos serviços digitais está o distanciamento físico das funções do prestador do serviço para um diálogo com o usuário e demais stakeholders; ampliando, assim, as 
possibilidades da coconstrução dos resultados do serviço e deixando de ser uma transferência unidirecional de informações (BALLANTYNE; NILSSON, 2017). Em função disso, os serviços digitais podem ser vistos como uma oportunidade para se desenvolver relacionamentos contínuos com os usuários, possibilitando a maior compreensão sobre os seus comportamentos, preferências, necessidades, entre outros, favorecendo a melhor adequação dos serviços.

As tentativas crescentes de personalizar e aumentar a transparência de processos em torno do serviço indicam um efeito positivo na ampliação da satisfação e confiança dos usuários (INBAR; TRACTINSKY, 2012). A compreensão acerca do que o usuário faz, como ele interage com os serviços e quais os fatores que o levam a tomar as decisões durante o processo podem gerar uma percepção crítica sobre o consumo desta mesma atividade em termos sociais, econômicos e ambientais (BHAMRA et al., 2016). Ao mesmo tempo, estas interações têm oferecido novas possibilidades de indução de comportamentos efetivamente mais sustentáveis, sendo as mesmas objeto de investigação da pesquisa reportada neste artigo.

\subsection{Definindo transparência na alimentação}

O termo transparência pode ter um significado diferente conforme a área de conhecimento, sendo um princípio amplo que tem epistemologia variada (PASQUIER; VILLENEUVE, 2007). De forma geral, a transparência implica abertura ou acesso (openness), comunicação (communication) e responsabilidade ética (accountability). A literatura sugere que para as organizações serem mais transparentes, torna-se necessário habilitar o acesso a informações na comunicação, assumir responsabilidade por suas ações, ter abertura a críticas e fornecer informações que possam ser comparadas aos padrões do setor (RAWLINS, 2008).

A transparência propicia o aprimoramento da capacidade de raciocínio dos públicos de uma organização e, ao mesmo tempo, contribui para responsabilização das organizações por suas ações, políticas e práticas. Neste sentido, a ampliação da transparência implica também na ampliação da confiança, pois à medida que as organizações se tornam mais transparentes, elas também se tornarão mais confiáveis (RAWLINS, 2008).

Sob a perspectiva deste estudo, o princípio da transparência é definido como sendo a habilidade de um produto, processo ou experiência alimentar em comunicar, a qualquer indivíduo, informações relevantes acerca das práticas sociais, ambientais e econômicas associadas direta ou indiretamente ao mesmo (WOGNUM et al., 2011; SCHIEFER; DEITERS, 2013).

Tal definição baseia-se no pressuposto de que a transparência é alcançada se todos os envolvidos na produção e no consumo de alimentos compreenderem as informações que são relevantes para a tomada de decisões, sendo estas válidas e confiáveis.

\subsection{Heurísticas para transparência no consumo de alimentos}

As expectativas em relação à transparência podem ter significados diferentes conforme atividade ou contexto do sistema alimentar (WOGNUM et al., 2011). Estas expectativas são 
influenciadas pela evolução dos estilos de vida, políticas públicas, escândalos alimentares, escassez de mercado, pelas características do valor provido pelo prestador de serviço e pela própria maturidade do consumidor (SCHIEFER; DEITERS, 2013).

Como se trata de um princípio voltado à comunicação, a eficácia da transparência vai depender da qualidade do formato, conteúdo e volume das informações transmitidas, assim como da capacidade de compreensão e motivação dos sujeitos receptores da informação. Isso implica que as informações devem ser relevantes, precisas, factuais, confiáveis, oportunas e disponíveis em uma quantidade apropriada (WOGNUM et al., 2011).

Dentre as categorias de informações utilizadas para transparência no setor de alimentos, destacam-se aquelas orientadas por regulamentações voltadas à fiscalização, à vigilância, à inspeção e ao controle de atributos de ordem técnica dos produtos alimentícios (SANTOS, 2011). Schiefer e Deiters (2013) as classificam em: segurança, qualidade e integridade alimentar.

Contudo, com o crescimento dos serviços digitais e correspondente evolução das expectativas e demandas do consumidor, observa-se a necessidade de adição de novos parâmetros na avaliação da transparência. É preciso melhor harmonia entre as demandas informacionais do consumidor e o perfil da informação requerida para se alcançar a transparência em serviços digitais.

A transparência no âmbito dos serviços digitais não se trata do fornecimento de um volume cada vez maior e indiscriminado de informações, mas, ao contrário, da customização da informação ao perfil e contexto de uso. Nesse sentido, as práticas de transparência usualmente adotadas no setor de alimentos para comunicação com os consumidores se concentram em cinco grupos (SANTOS, 2009; SCHIEFER; DEITERS, 2013):

- Transparência dos impactos ambientais

- Transparência dos aspectos sociais

- Transparência dos processos de produção

- Transparência na manipulação de informações

- Transparência para formação dos consumidores

Além destas práticas, a transparência no setor de alimentos também é influenciada pelo background cultural, os costumes locais e localização da produção. Esses fatores impactam diretamente na qualidade e adequação das informações possíveis e passíveis de serem oferecidas aos consumidores. Como consequência, as práticas formais de transparência podem diferir significativamente entre regiões e culturas (SCHIEFER; DEITERS, 2013).

\section{Método de Pesquisa}

Considerando a característica exploratória do problema de pesquisa e o objetivo de propor um modelo teórico para orientar o diagnóstico da transparência em serviços, foi selecionado o método de pesquisa Design Science Research (DSR) (DRESCH et al., 2015; SANTOS, 2018). De acordo com Dresch et al. (2015), "modelos" podem ser entendidos como um artefato caracterizado por conter um conjunto de proposições ou declarações que expressam as relações entre conceitos. 
A Design Science Research é um método de pesquisa que alia a teoria à prática, indicado quando o objetivo do estudo é projetar e desenvolver artefatos, bem como soluções prescritivas, para gerar conhecimentos que suportem a solução de problemas (DRESCH et al., 2015; SANTOS, 2018).

A aplicação do método foi organizada em três fases com base em Dresch et al. (2015), conforme ilustra a Figura 1, contemplando os procedimentos adotados e seus respectivos resultados.

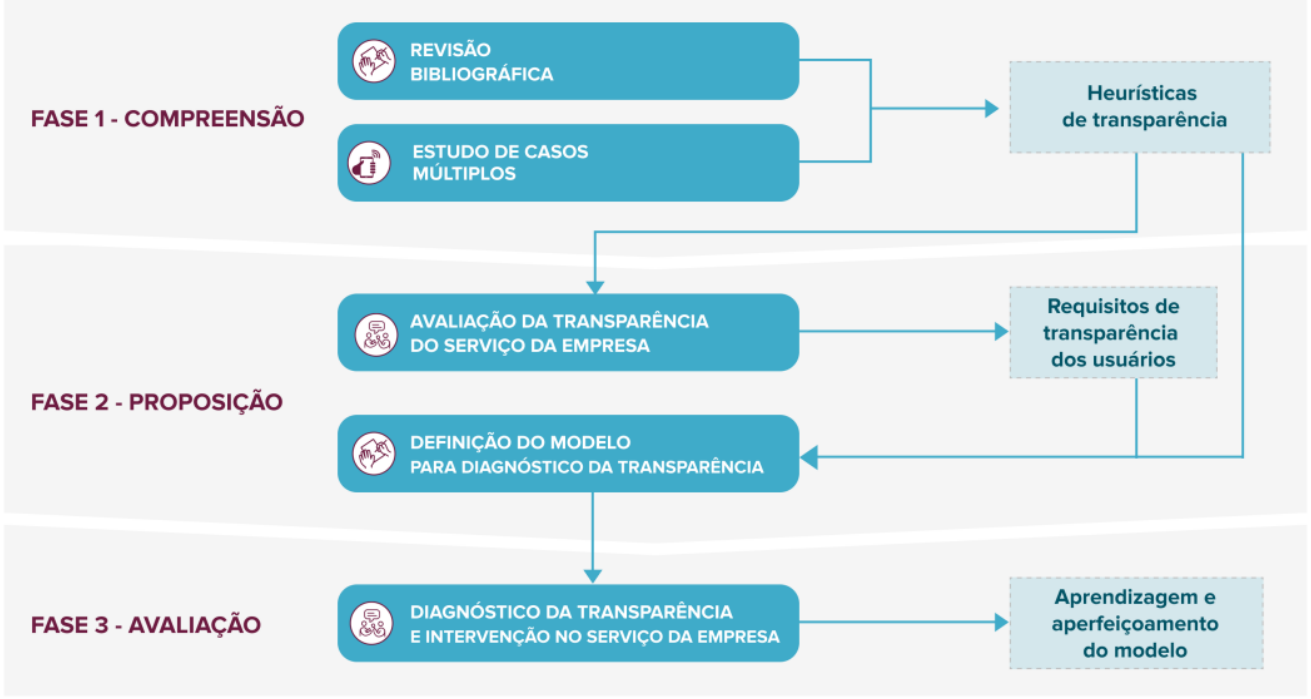

Figura 1: Visão geral da estratégia da pesquisa. Fonte: os autores.

\subsection{Método de Pesquisa - Fase 1 de Compreensão}

A primeira fase da pesquisa trata da compreensão do problema e segue uma lógica mais indutiva, precedendo a aplicação da lógica abdutiva da Fase 2. Buscou-se a compreensão sistêmica do problema a partir de múltiplas fontes de dados. Inicialmente, para compreender a perspectiva da teoria, foi conduzida uma Revisão Bibliográfica assistemática e sistemática dos temas abordados, buscando levantar princípios e heurísticas associados à transparência em serviços digitais. Em seguida, foi realizado um Estudo de Casos Múltiplos (ex-post-facto) para confrontar as heurísticas de base teórica, com as principais características das soluções disponíveis no mercado. Buscou-se identificar as heurísticas nesses casos via Teoria Fundamentada nos Dados (Grounded Theory), na qual são realizadas inferências sobre os dados através de abstração do significado via ciclos sucessivos e cumulativos de codificação e categorização (SANTOS, 2018).

A seleção dos casos considerou tanto empresas startups (empresas jovens) conhecidas como food techs, quanto empresas já estabelecidas, tendo em conta que ambas implementam soluções, de base tecnológica digital, que podem influenciar as decisões de consumo de alimentos quanto a saúde e/ou sustentabilidade. A integração dos resultados do Estudo de Casos Múltiplos com a Revisão Bibliográfica possibilitou a identificação de um conjunto de heurísticas para o diagnóstico da transparência em serviços digitais. 


\subsection{Método de Pesquisa - Fase 2 de Proposição do Modelo}

Para o desenvolvimento do modelo teórico para diagnóstico da transparência, selecionou-se uma empresa que prestava um serviço digital no setor, voltado ao consumo de alimentos orgânicos e situada em Curitiba-PR. Foi realizado o mapeamento e o diagnóstico do estado da transparência no serviço atual, tendo como informantes chave os sócios-fundadores da empresa. Para avaliação preliminar da transparência do serviço, foi aplicado um checklist com base nas heurísticas identificadas a partir da Revisão Bibliográfica e dos múltiplos estudos de caso.

Esta fase envolveu a aplicação do Design Etnográfico na busca por subsídios para a concepção do modelo, contribuindo para a compreensão do nível de transparência e identificação das possibilidades para se ampliar a transparência, na percepção dos usuários. Para a seleção dos usuários na etapa etnográfica, foram desenvolvidas Personas a partir da Revisão Bibliográfica, com ênfase em perfis associados à alimentação sustentável, utilizando as proposições de Verain et al. (2012). O desenvolvimento das Personas também levou em conta características sociodemográficas e fatores que influenciam o consumo de alimentos referentes à população brasileira, inclusive, quanto aos alimentos orgânicos, conforme proposições de Caisan (2016) e Organis (2019). As ferramentas utilizadas para coleta de dados foram baseadas em sondas culturais.

A partir dos resultados desta etapa etnográfica, foi realizada a formalização dos requisitos de transparência dos usuários, através da análise individual e cruzamento dos dados coletados junto aos participantes. Os resultados foram apresentados à empresa parceira em um workshop nas dependências da universidade, em que se buscou compreender a percepção da empresa quanto às possibilidades de atendimento dos requisitos identificados.

Estes resultados possibilitaram a proposição do artefato "Modelo teórico para diagnóstico da transparência em serviços digitais do setor de alimentos". A proposição do modelo utilizou como base informacional a convergência das heurísticas coletadas na Fase 1, incluindo as identificadas na teoria e nos estudos de caso múltiplos, junto dos requisitos dos usuários. A partir da organização e codificação das heurísticas, foi realizada a formalização dos conceitos gerais utilizados na proposição do modelo, sendo eles: a) Dimensões ou tipos de transparência que podem ser habilitadas de acordo seu potencial de impacto para o serviço; b) Fatores que qualificam cada dimensão da transparência; c) Heurísticas ou práticas de transparência em serviços.

O modelo proposto busca tanto o apoio ao diagnóstico de serviços digitais existentes, como o apoio às atividades de criação de soluções para o aumento da transparência em serviços digitais.

\subsection{Método de Pesquisa - Fase 3 de Avaliação}

A última fase da pesquisa tratou da avaliação do modelo proposto, através de sua aplicação na empresa parceira. Essa avaliação ocorreu através de sua aplicação no diagnóstico e projeto de intervenção no serviço digital investigado. Assim como na Fase 1, a avaliação utilizou como 
principais critérios a viabilidade das proposições oriundas da aplicação do modelo, assim como o grau de inovação e atratividade das mesmas. Estes resultados foram apresentados para a empresa em formato de workshop nas dependências da universidade, e através de encontros individuais e presenciais no caso dos usuários. Os resultados dessa fase possibilitaram a identificação de aperfeiçoamento no modelo, sendo que o presente artigo reporta o resultado desse processo.

\section{Resultados}

\subsection{Resultados da Fase 1 de Compreensão}

A partir da Revisão Bibliográfica, principalmente das proposições de Pasquier e Villeneuve (2007), Rawlins (2008), Wognum et al. (2011) e Schiefer e Deiters (2013), foram identificadas cinco classes de heurísticas: Transparência dos impactos ambientais; Transparência dos aspectos sociais; Transparência dos processos de produção; Transparência na manipulação de informações e Transparência para formação do consumidor. Estas classes de heurísticas contribuíram para a identificação de estudos de caso relevantes, onde a prática da transparência estava presente nos serviços digitais.

Na etapa de Estudo de Casos Múltiplos foram selecionadas oito empresas com oferta de serviço digital no setor de alimentos, classificadas em quatro categorias: Entrega de alimentos da fazenda à mesa (Farm to table food delivery); Rastreabilidade da cadeia de alimentos (Food supply chain traceability); Alimentação saudável, nutrição e refeições (Healthy eating, nutrition and meals) e Agricultura como serviço (Farming as a service). Através da aplicação da Grounded Theory, novas heurísticas foram identificadas ao longo do processo de análise desses casos. Desta forma, foram mapeadas vinte e seis heurísticas teórico-práticas de transparência, que serviram para instrumentalizar a versão inicial do modelo proposto (NICASTRO, 2020).

O cruzamento entre a fundamentação teórica e os múltiplos Estudos de Caso possibilitou a identificação das principais ênfases da aplicação das heurísticas para ampliação da transparência em: Transparência sobre os processos de produção; Transparência na manipulação das informações e Transparência para formação dos consumidores.

\subsection{Resultados da Fase 2 de Proposição do Modelo}

Para realizar o desenvolvimento da proposição do "modelo", foi selecionada uma empresa do segmento de "Assinatura para entrega de alimentos orgânicos", similar à categoria de empresas nomeada como "Entrega de alimentos da fazenda à mesa" (Farm to table food delivery). A empresa tem sua sede em Curitiba-PR, com tempo de operação de cerca de um ano na época da pesquisa. A Figura 2 mostra o Mapa de Sistema do serviço da empresa. Nota-se que a empresa atua como um intermediário entre produtores locais e o consumidor final, mas inclui, também, atacadistas para o caso de produtos orgânicos não encontrados localmente. 


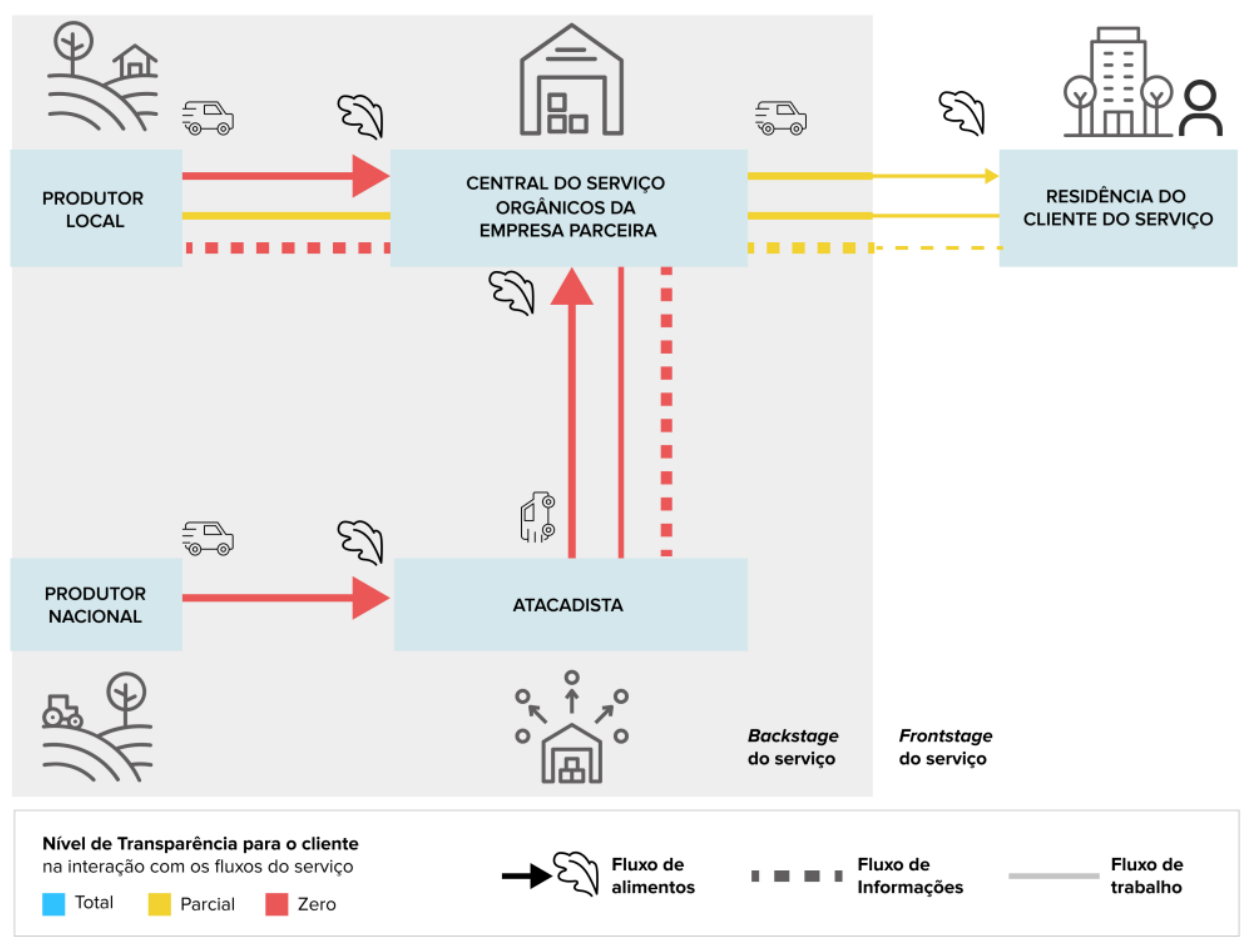

Figura 2: Exemplo do Mapa de Sistema do serviço da empresa parceira da pesquisa. Fonte: os autores.

Esse mapeamento permitiu identificar uma maior concentração dos fluxos de trabalho, alimentos e informações que compõem a operação da empresa nos bastidores (backstage) do serviço. Nota-se, porém, que o cliente final tinha pouco acesso e visibilidade acerca destes fluxos e suas respectivas informações na sua interação por meio dos pontos de contato disponíveis. Além disso, também foi possível identificar, nos bastidores do serviço, um volume de fluxos e informações superiores aos que eram disponibilizados aos clientes.

Os dados coletados mostraram a elevada competência por parte dos sócios da empresa acerca da teoria e prática associada à produção e ao consumo sustentável de alimentos. No entanto, de acordo com os resultados da avaliação inicial, a empresa parceira atendia parcialmente as heurísticas de transparência mapeadas preliminarmente.

Até o início da pesquisa, o tema "transparência" não fazia parte de uma temática central ao planejamento estratégico ou tático da empresa. A entrevista realizada no início do projeto mostrou que a transparência era compreendida como comunicação com o cliente e, também, rastreabilidade das informações. Nesta entrevista, ficou claro que a transparência era entendida como uma estratégia de fidelização dos clientes ao serviço da empresa.

A pesquisa com os usuários, via Design Etnográfico, ocorreu com uma amostra total de seis participantes incluindo clientes e não clientes do serviço da empresa parceira, sendo: duas participantes representantes do perfil "Consumidora Convencional"; duas participantes representantes do perfil "Consumidora Saúde"; duas participantes representantes do perfil "Consumidora Consciente".

Apesar do escopo da pesquisa ter como recorte as atividades relacionadas à aquisição de alimentos, a partir dos registros e relatos das participantes do estudo etnográfico, identificou-se que os momentos da jornada de usuário mais críticos para a ampliação da transparência eram: a) 
planejamento da alimentação e das compras; b) realização das compras ou contratação do serviço e c) preparo e consumo das refeições.

Ao final, nesta etapa etnográfica, foram especificados vinte e três requisitos de transparência sob a perspectiva dos usuários participantes, agrupados conforme os temas de afinidade listados a seguir: a) Manipulação das informações do serviço; b) Credibilidade do serviço; c) Formas de ingresso ao serviço; d) Opções para personalização do serviço; e) Relação dos usuários com a alimentação através do serviço e f) Consumo consciente de alimentos através do serviço.

A partir destes resultados, as percepções e necessidades de transparência expressadas pelos usuários permitiram identificar requisitos para o aperfeiçoamento de serviços digitais, requisitos estes não explicitamente declarados na literatura ou nos múltiplos estudos de caso analisados inicialmente.

A partir destas etapas, formulou-se o modelo proposto, o qual integra elementos da Revisão Bibliográfica sobre a transparência no setor de alimentos (RAWLINS, 2008; WOGNUM et al., 2011; SCHIEFER; DEITERS, 2013; EAKIN et al., 2017), dos Estudos de Caso Múltiplos e, também, da investigação preliminar realizada na empresa parceira, reportada nesta seção.

A criação do modelo partiu do pressuposto de que a transparência em serviços digitais poderia seguir uma lógica de progressiva evolução. A estrutura do modelo é formada pelos grupos de heurísticas identificados na Fase 1. O modelo propõe três dimensões para avaliação da transparência. Estas dimensões variam suas definições com base no nível de envolvimento do usuário, conforme ilustra a Figura 3.

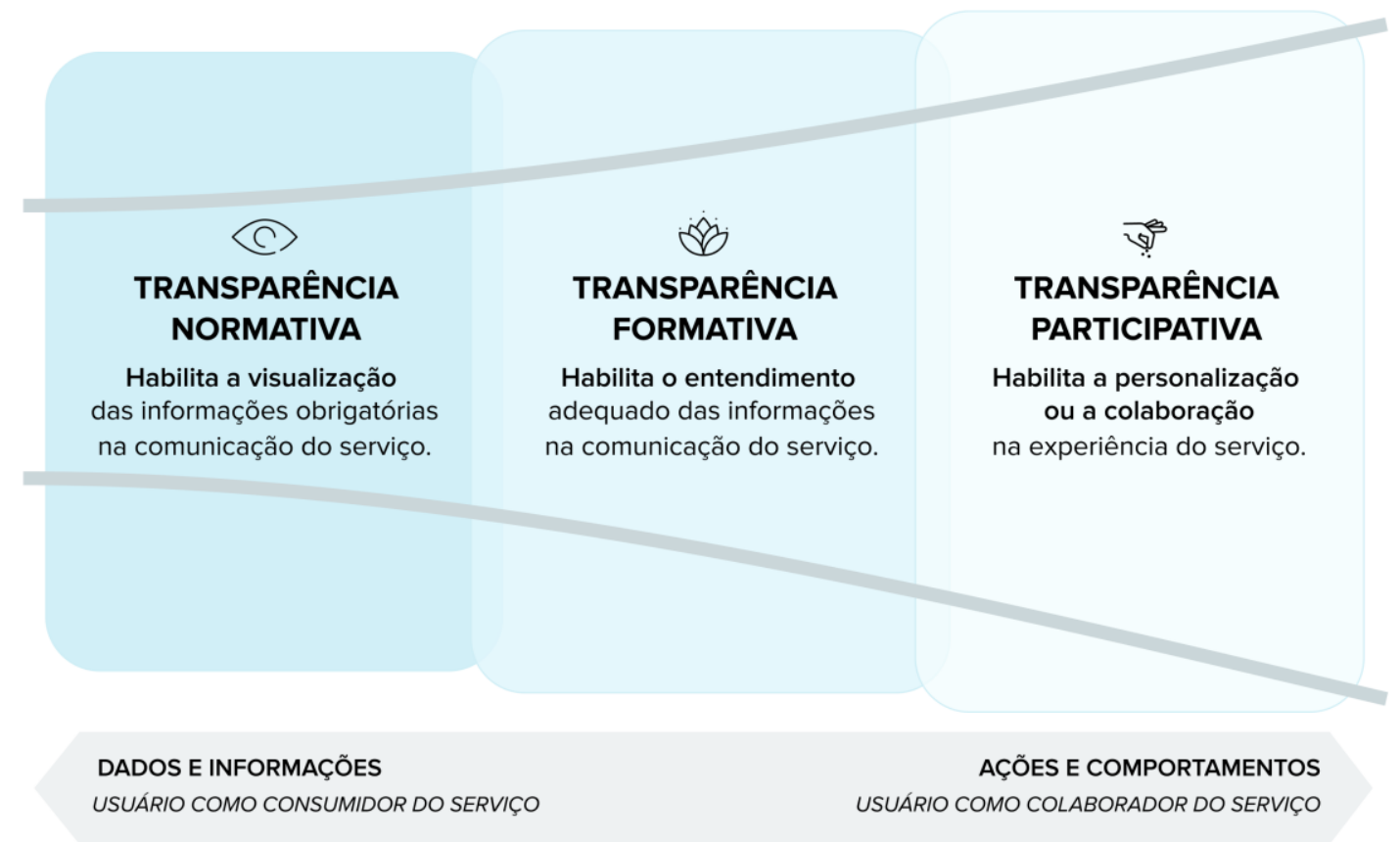

Figura 3: Modelo teórico para diagnóstico da transparência em serviços digitais. Fonte: os autores.

A seguir, são descritas cada uma das dimensões do modelo proposto: 


\subsubsection{Dimensão 1 - Transparência Normativa}

A dimensão Normativa do modelo proposto permite a visualização dos dados e/ou informações, caracterizados principalmente por aqueles exigidos no atendimento a normas e regulamentações específicas do setor de alimentos. Geralmente é a primeira estratégia de transparência que as organizações adotam. O Quadro 1 apresenta os fatores e heurísticas associadas a esta dimensão.

\begin{tabular}{|l|l|}
\hline Fatores & Heurísticas \\
\hline Disponibilidade & Permite o acesso aos dados e informações obrigatórias \\
\hline Integridade & Indica a presença de alérgenos e/ou outros ingredientes passíveis de restrições \\
\hline Verificabilidade & Garante que as informações são consistentes, sem ruídos, perdas, atrasos ou distorção \\
\hline & Fornece indicação de conformidade com padrões de qualidade verificáveis \\
\hline & Faz uso de selos de sistemas de certificação \\
\hline & Permite verificar as informações fornecidas \\
\hline
\end{tabular}

Quadro 1: Dimensão Normativa do Modelo para diagnóstico da transparência em serviços digitais. Fonte: os autores.

A partir dos resultados da Fase 1 da pesquisa, identificou-se que esta dimensão da transparência pode contribuir para a visibilidade das informações referentes a uma gama maior de elementos envolvidos na entrega de um serviço digital (ex: pontos de contato, stakeholders, processos etc.), indo além das informações que caracterizam e que são incorporadas junto ao alimento como produto.

Comparando tais práticas com as expectativas dos usuários junto à empresa parceira, entendese que as estratégias baseadas apenas na transparência normativa podem não atender completamente às necessidades de transparência de um serviço. Os usuários podem não possuir os mecanismos necessários para interpretar adequadamente os dados disponíveis, ignorando ou estabelecendo suposições falsas. Além disso, ainda que as informações tenham o formato, quantidade e momento adequado de exposição, o usuário pode ter um baixo interesse em seu conteúdo. Para tanto, faz-se necessário a aplicação da dimensão formativa, conforme descrito a seguir.

\subsubsection{Dimensão 2 - Transparência Formativa}

A dimensão Formativa do modelo proposto visa capacitar o usuário para que a transparência seja efetiva. Enfatiza o desenvolvimento de conhecimento e habilidades que possibilitem a interpretação e o entendimento adequados em relação aos dados e informações fornecidas ao longo do serviço. Contribui, desta forma, para sensibilizar e capacitar o usuário sobre ter uma alimentação saudável e sobre a produção e o consumo mais sustentável dos alimentos. O Quadro 2 apresenta os fatores e heurísticas associadas a esta dimensão da transparência. 


\begin{tabular}{|c|c|}
\hline Fatores & Heurísticas \\
\hline \multirow[t]{7}{*}{ Conscientização } & Esclarece o posicionamento do serviço com relação à sustentabilidade na alimentação \\
\hline & Diferencia a comunicação dos alimentos de manuseio justo e sustentável dos produtores \\
\hline & Evidencia as informações e os indicadores a respeito do impacto ambiental do serviço \\
\hline & Converte parâmetros invisíveis em visíveis \\
\hline & $\begin{array}{l}\text { Torna acessíveis os métodos adotados para o cálculo de indicadores e manipulação dos } \\
\text { dados }\end{array}$ \\
\hline & Promove os benefícios nutricionais dos alimentos \\
\hline & Proporciona a descoberta de alimentos não habituais \\
\hline \multirow[t]{9}{*}{ Compreensão } & Auxilia na interpretação e entendimento das informações \\
\hline & Promove o aprendizado sobre os alimentos \\
\hline & $\begin{array}{l}\text { Apresenta os critérios ético-sociais adotados, envolvendo os stakeholders, cultura e } \\
\text { recursos }\end{array}$ \\
\hline & Permite o rastreamento da cadeia produtiva dos alimentos do serviço \\
\hline & Orienta sobre as atitudes que contribuem para o consumo mais sustentável \\
\hline & Esclarece o funcionamento do serviço em todas as suas etapas / situações de uso \\
\hline & Integra controles sensoriais aos produtos e processos associados aos alimentos \\
\hline & $\begin{array}{l}\text { Permite a direta visualização de todos os elementos relevantes ao alimento e seus processos } \\
\text { associados }\end{array}$ \\
\hline & Promove visitas, apresentações e/ou demonstrações no local, com explicações detalhadas \\
\hline \multirow[t]{5}{*}{ Utilidade } & Adequa as informações conforme as necessidades dos usuários e seus contextos de uso \\
\hline & Fornece indicação de histórico de frescor dos alimentos oferecidos \\
\hline & Disponibiliza uma plataforma educacional sobre alimentação saudável e sustentável \\
\hline & $\begin{array}{l}\text { Disponibiliza instruções para os alimentos oferecidos quanto ao preparo, consumo, } \\
\text { conservação e descarte }\end{array}$ \\
\hline & $\begin{array}{l}\text { Adequa benefícios e conveniência do serviço conforme necessidades dos usuários e seus } \\
\text { momentos de vida }\end{array}$ \\
\hline
\end{tabular}

Quadro 2: Dimensão Formativa do Modelo para diagnóstico da transparência em serviços digitais. Fonte: os autores.

A pesquisa junto aos usuários com a empresa parceira sugere que estratégias baseadas apenas em transparência formativa podem ter como barreira a falta de interesse e/ou capacidade cognitiva dos usuários para lidar com as informações. Diferentes necessidades, comportamentos, contextos e prioridades de informações podem ser demasiadamente desafiadores e pouco relevantes para $\mathrm{o}$ usuário. Ainda, as empresas podem ter receio de divulgar informações que possam beneficiar os concorrentes. Assim, a transparência necessita ser compreendida como uma meta móvel, em contínua melhoria à medida que o usuário e a organização evoluem em sua maturidade no tema. Alcançar tal evolução não depende apenas da dimensão formativa, exigindo o engajamento ativo dos usuários, ou seja, a aplicação da dimensão participativa descrita a seguir.

\subsubsection{Dimensão 3 - Transparência Participativa}

A dimensão Participativa do modelo proposto inclui o rol de heurísticas que tratam do engajamento do usuário (assim como todos dos stakeholders envolvidos no serviço), permitindo um nível de transparência a partir do qual os usuários possam personalizar (para si) e contribuir (para a comunidade) com a melhoria contínua da experiência com o serviço. Em função das características da transparência formativa, infere-se que sua aplicação seja mais provável e 
possível em serviços que são mais centrados nos usuários. O Quadro 3 apresenta os fatores e heurísticas associadas a esta dimensão da transparência.

\begin{tabular}{|c|c|}
\hline Fatores & Heurísticas \\
\hline \multirow[t]{2}{*}{ Usabilidade } & Facilita o uso e a interação dos usuários com os instrumentos de informação do serviço \\
\hline & Facilita o uso e a interação dos usuários com os processos e atividades do serviço \\
\hline \multirow[t]{5}{*}{ Controle } & $\begin{array}{l}\text { Permite que os usuários interajam com informações do serviço por meio de colaboração ou } \\
\text { compartilhamento }\end{array}$ \\
\hline & Permite aos usuários o controle sobre as decisões alimentares acerca da oferta do serviço \\
\hline & Fornece informações personalizadas para os usuários \\
\hline & Permite que os usuários experimentem o serviço sem compromisso de fidelização \\
\hline & Permite que os usuários estabeleçam uma relação de coparticipação na entrega do serviço \\
\hline \multirow[t]{4}{*}{ Engajamento } & Atende os usuários de forma ágil e atenciosa \\
\hline & $\begin{array}{l}\text { Disponibiliza um canal interativo para atendimento e relacionamento contínuo com os } \\
\text { usuários }\end{array}$ \\
\hline & Demonstra conhecimento sobre o perfil, interesses e restrições dos usuários \\
\hline & Permite o contato direto com uma pessoa responsável pela entrega do serviço \\
\hline
\end{tabular}

Quadro 3: Dimensão Participativa do Modelo para diagnóstico da transparência em serviços digitais. Fonte: os autores

Os serviços digitais oferecem ampla gama de oportunidades para permitir que organizações possibilitem a participação dos usuários. Estas oportunidades implicam transformar a função do usuário, transformando sua posição de mero consumidor passivo para que possa assumir o papel de um efetivo colaborador no serviço. O usuário pode até mesmo se tornar um ativista em prol de uma alimentação mais sustentável.

Estratégias baseadas apenas na transparência participativa podem ter como barreiras o desinteresse por interações ativas e/ou limitações na capacidade cognitiva dos usuários. Similarmente, as limitações incluem os próprios limites culturais e estratégicos das organizações quanto ao nível de envolvimento com os usuários. O receio da organização ser desafiada quanto às suas práticas pode reduzir o interesse em manter um diálogo aberto com os usuários (PASQUIER; VILLENEUVE, 2007).

\subsection{Resultados da Fase 3 - Avaliação}

Esta fase tratou da avaliação do modelo proposto, sendo utilizado para tal um protótipo conceitual criado para representar as intervenções de ampliação de transparência no serviço atual da empresa parceira da pesquisa. O diagnóstico foi aplicado em sessão presencial e individual junto aos usuários participantes da Fase 2 da pesquisa. Primeiramente, foi realizada a apresentação do protótipo do serviço. Em seguida, foi solicitado que o usuário descrevesse a transparência percebida, assim como a transparência considerada desejada (ou suficiente), tendo como critérios o checklist baseado nas dimensões, fatores e heurísticas do modelo proposto.

Os resultados foram consolidados em um diagrama do tipo aranha, conforme ilustra a Figura 4. Neste diagrama, são apresentados os resultados da aplicação do diagnóstico na versão original implementada do serviço da empresa parceira, em comparação com a versão do 
conceito/protótipo desenvolvido com base no modelo proposto. Ambas têm como ponto de partida as percepções de transparência dos usuários do serviço.

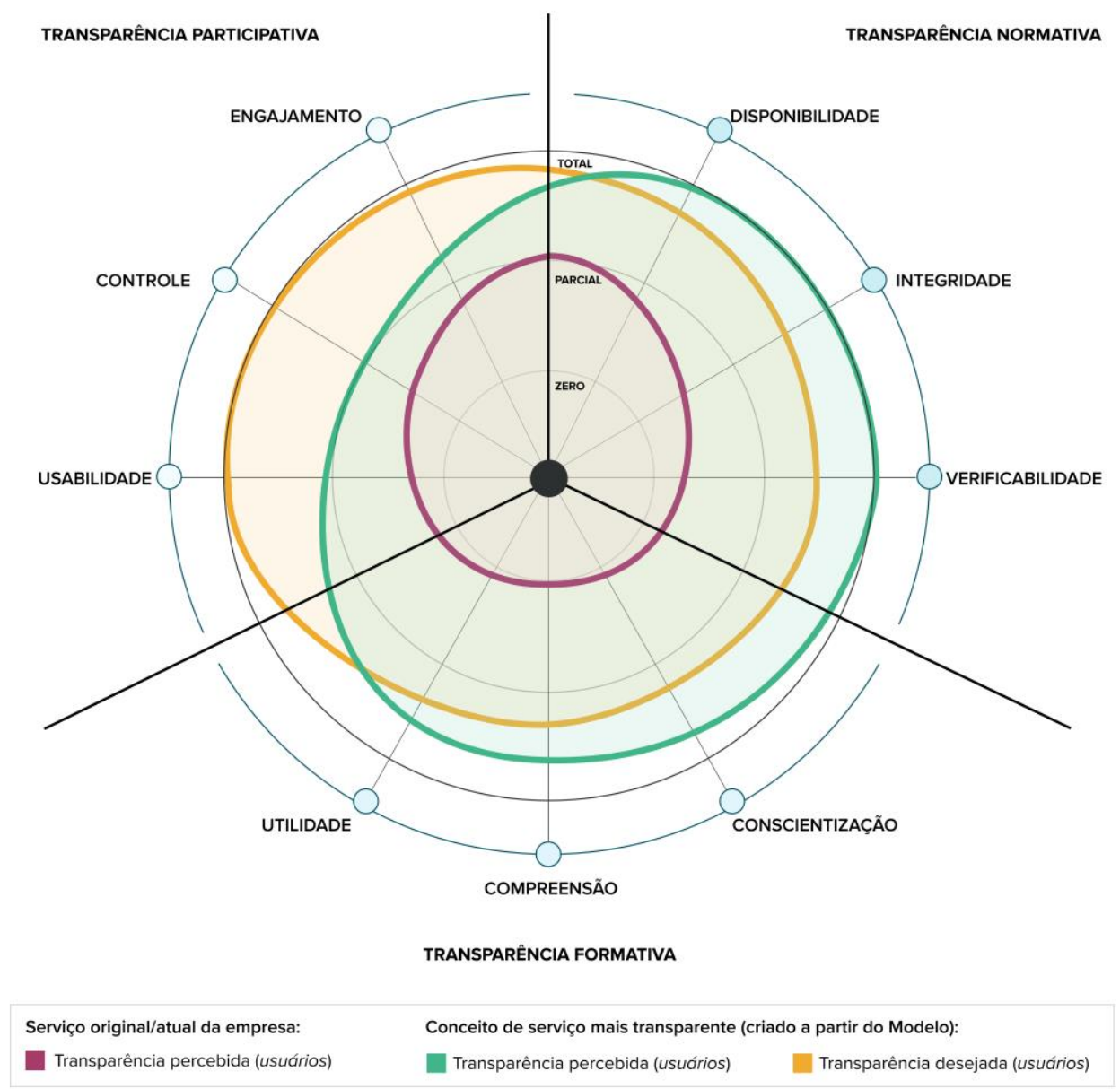

Figura 4: Diagnóstico da transparência do serviço da empresa a partir do Modelo proposto. Fonte: os autores.

Conforme já identificado preliminarmente na pesquisa, o serviço vigente da empresa parceira não atendia suficientemente os requisitos de transparência dos usuários quanto às dimensões formativa e participativa. As intervenções projetadas para o protótipo do serviço, com base no modelo proposto, permitiram identificar a adequação do seu conceito para ampliar a transparência em todas as dimensões do modelo.

O diagnóstico foi apresentado e discutido junto dos sócios representantes da empresa parceira, sendo reforçada a necessidade de capacitar os usuários para que estes percebam e compreendam os valores dos alimentos orgânicos e, consequentemente, dos serviços associados (ou seja, a dimensão formativa). A Fase 2 mostrou que anteriormente a empresa partia do pressuposto que os usuários já sabiam e entendiam o que lhes era oferecido. Tal pressuposto ocorria principalmente pelo negócio ter nascido em feiras, e depois ter migrado para o ambiente digital. No entanto, ficou claro que em um ambiente mediado por interfaces digitais, o serviço precisa fornecer os mecanismos para habilitar a comunicação e o comportamento dos usuários de forma transparente e contínua, configurando-se como um espaço permanente de aprendizado. 


\section{Considerações finais}

A partir dos resultados da pesquisa, foi proposto um modelo teórico para apoiar o diagnóstico da transparência em serviços já existentes, além do processo criativo de aperfeiçoamento e/ou o desenvolvimento de novos serviços do setor de alimentos.

O modelo proposto pode ser instrumental na concepção de protocolos e ferramentas voltadas à melhoria da transparência em serviços digitais. Quanto à sua utilidade, os autores sugerem a sua utilização como uma ferramenta complementar ao processo de Design de Serviço, no escopo do conceito geral do serviço, ou no escopo de jornadas de experiência do usuário. Mais especificamente, pode ser utilizado em atividades de diagnóstico, mapeamento de oportunidades, ideação, conceituação e prototipação, além de planejamento de negócio.

Em pesquisas voltadas ao desenvolvimento de protocolos e ferramentas associadas ao modelo proposto, os autores sugerem a utilização da lista de heurísticas desenvolvida na forma de um checklist de transparência. Posteriormente a esta coleta, os dados podem ser transpostos para o diagrama do tipo aranha, como ferramenta de visualização dos resultados do diagnóstico, conforme foi empregado no estudo de campo.

A literatura sobre design para transparência se encontra em um estágio inicial do conhecimento, enfatizando estratégias voltadas para a comunicação e rastreabilidade de informações ainda restritas ao nível de artefatos físicos. No entanto, os resultados desta pesquisa mostraram que o conhecimento existente sobre transparência já se encontra consolidado o suficiente para conceber ou reformular a proposta de valor dos serviços, expandindo as possibilidades de uso e interação para usuários e stakeholders.

Ampliar a transparência pode ter repercussões para além do escopo dos serviços digitais, afetando outras dimensões do negócio. Dependendo dos objetivos de transparência da empresa, pode ser necessário intervir nos canais e pontos de contato do serviço. Quanto mais as necessidades de transparência caminharem para além do sinônimo de uma mera apresentação de informações exigidas por lei, maior é o potencial de impacto sobre a influência de um serviço na mudança de comportamentos na direção de um consumo mais sustentável.

Por fim, apesar da complexidade de informações e interações que envolve uma perspectiva mais sistêmica para transparência, os autores recomendam que as estratégias não tomem como ponto de partida apenas as novas possibilidades tecnológicas digitais. A utilização dos princípios para a sustentabilidade como foco da melhoria da transparência exemplifica tal abordagem.

\section{Referências}

BALLANTYNE, David; NILSSON, Elin. All that is solid melts into air: the servicescape in digital service space. Journal of Services Marketing, Bingley, v. 31, n. 3, p. 226-235, 2017.

BHAMRA, Tracy; LILLEY, Debra; TANG, Tang. Design for Sustainable Behaviour: Using Products to Change Consumer Behaviour. The Design Journal, Oxfordshire, v. 14, n. 4, p. 427-445, 2016. 
BUCHANAN, Richard. Design Research and the New Learning. Design Issues, Cambridge (EUA), v. 17, n. 4, p. 3-23, 2001.

CAISAN (CÂMARA INTERSETORIAL DE SEGURANÇA ALIMENTAR E NUTRICIONAL). Primeiro Plano Municipal de Segurança Alimentar e Nutricional de Curitiba: 2016/2019. Curitiba, 2016. 164p.

COSTA, Filipe.; YUDI, Ricardo. Um ensaio sobre a etologia do Food Design. Revista Latinoamericana de Food Design, Porto Alegre, v. 1, n. 1, p. 1-134, 2020.

DRESCH, Aline; LACERDA, Daniel Pacheco; ANTUNES, José Antônio Valle. Design Science Research: método de pesquisa para avanço da ciência e tecnologia. Porto Alegre: Bookman Editora, 2015. 204p.

EAKIN, Hallie et al. Identifying attributes of food system sustainability: emerging themes and consensus. Agriculture and Human Values, New York, v. 34, n. 3, p. 757-773, 2017.

FREIRE, Karine de Mello; DAMAZIO, Vera. Anais do 9o Congresso Brasileiro de Pesquisa e Desenvolvimento em Design. São Paulo: Blücher e Universidade Anhembi Morumbi, 2010. $9 \mathrm{p}$.

HARTWIG, Katharina; BILLERT, Matthias Simon. Measuring Service Quality: A Systematic Literature Review. In: European Conference on Information Systems (ECIS), Portsmouth, 2018.

INBAR, Ohad; TRACTINSKY, Noam. Lowering the line of visibility: Incidental users in service encounters. Behaviour and Information Technology, Abingdon, v. 31, n. 3, p. 245260, 2012.

MORITZ, Stefan. Service Design: practical access to an evolving field. 2005. 246p. Dissertação (Mestrado em Design) - Köln International School of Design, Cologne, 2005.

NICASTRO, Marcella Lomba. Modelo para diagnóstico da transparência em serviços digitais para o consumo mais sustentável de alimentos. 2020. 209 p. Dissertação (Mestrado em Design) - Programa de Pós-graduação em Design, Universidade Federal do Paraná, Curitiba.

ORGANIS (ASSOCIAÇÃO DE PROMOÇÃO DOS ORGÂNICOS). Panorama do consumo de orgânicos no Brasil. Curitiba, 2019. 69p.

OSTROM, Amy. L. et al. Service Research Priorities in a Rapidly Changing Context. Journal of Service Research, Thousand Oaks, v. 18, n. 2, p. 127-159, 2015.

PASQUIER, Martial; VILLENEUVE, Jean-Patrick. Organizational barriers to transparency: A typology and analysis of organizational behavior tending to prevent or restrict access to information. International Review of Administrative Sciences, Thousand Oaks, v. 73, n. 1, p. 147-162, 2007.

PENIN, Lara. An Introduction to Service Design: Designing the Invisible. London: Bloomsbury Publishing, 2017. 334p. 
POUTANEN, Kaisa. et al. Food Economy 4.0: VTT's vision of an era of smart consumercentric food production. Finland: VTT Technical Research Centre of Finland Ltd., 2017. 60p.

RAWLINS, Brad. Measuring the relationship between organizational transparency and employee trust. Public Relations Journal, Amsterdam, v. 2, n. 2, p. 1-21, 2008.

SANGIORGI, Daniela; PRENDIVILLE, Alison; RICKETTS, Amy. Mapping and developing service design research in the UK. London: Service Design Research UK Network, 2014. 41p.

SANTOS, Aguinaldo. Gerenciamento visual de sistemas de produção: Teoria e prática. Curitiba: Custom Books Publishing, 2009. 114p.

SANTOS, Aguinaldo. Seleção de Método de Pesquisa: guia para pós-graduandos em design e áreas afins. Curitiba: Ed. Insight. 2018. 230p.

SANTOS, Célio Barbosa. Segurança alimentar e rotulagem de alimento sob a perspectiva do código de defesa do consumidor brasileiro. Percurso Acadêmico, Belo Horizonte, v. 1, n. 1, p. 327-346, 2011.

SCHIEFER, Gerard; DEITERS, Jivka. Transparency in the Food Chain. Bonn: Universität Bonn-ILB Press, 2013. 371 p.

VERAIN, Muriel et al. Segments of sustainable food consumers: A literature review. International Journal of Consumer Studies, New Jersey, v. 36, n. 2, p. 123-132, 2012.

WEF (WORLD ECONOMIC FORUM). Innovation with a Purpose: The role of technology innovation in accelerating food systems transformation. Geneva, 2018. 42p.

WOGNUM, Nel et al. Systems for sustainability and transparency of food supply chains current status and challenges. Advanced Engineering Informatics, Amsterdam, v. 25, n. 1, p. 65-76, 2011.

\section{Sobre os autores}

\section{Marcella Lomba Nicastro}

Doutoranda em Design pela Universidade Federal do Paraná - Brasil, junto ao Núcleo de Pesquisa em Design e Sustentabilidade. Sua pesquisa explora a interseção da transparência para a sustentabilidade e o design de serviço. Possui mestrado em Design pela UFPR (2020), especialização em Design Centrado no Usuário pela Universidade Positivo (2013) e graduação em Engenharia de Computação pela PUC-Campinas (2006).

https://orcid.org/0000-0003-3810-7041

\section{Aguinaldo dos Santos}

Mecânico de Automóveis (SENAI/1984), Engenheiro Civil (UFPR/1992), MSc em Engenharia Civil (UFRGS/1995), PhD - Built Environment (SALFORD/Inglaterra/1999), Pós-doutorado em Design para a Sustentabilidade (POLIMI/2010). Bolsista Produtividade do CNPq. Coordenador do Núcleo de Design \& Sustentabilidade e Professor no Curso de Design de Produto e Programa de Pós-Graduação em Design da UFPR.

https://orcid.org/0000-0002-8645-6919 